\title{
EL PERIMENTO BRAQUIAL EN LA MUJER GESTANTE, Y SU RELACIÓN CON OTRAS MEDIDAS ANTROPOMÉTRICAS MATERNAS*
}

\section{MID ARM CIRCUMFERENCE IN PREGNANT WOMEN AND ITS RELATION TO OTHER MATERNAL ANTHROPOMETRIC INDICATORS}

Anamaria E. Ricalde

Arnaldo A. F: de Siqueira ${ }^{2}$

RICALDE, A. E.; SIQUEIRA, A. A. F. de. El Perimetro Braquial en la Mujer Gestante, y su Relación con otras Medidas Antropométricas Maternas. Rev. Bras. Cresc. Desenv. Hum., São Paulo, 6 (1/2), 1996.

\begin{abstract}
Resumen: Con el objetivo de determinar las variaciones dei perimetro braquial durante ei embarazo y verificar la relación entre este indicador y otras medidas antropométricas, fue realizado un estúdio de corte transversal y de seguimeinto de 200 mujeres embarazadas.Fueron medidas en cada mujer: peso, perimetro braquial, altura uterina, altura, y peso pre gestacional. Para ei análisis de los resultados las mujeres fueron agrupadas por trimestre del embarazo. La diferencia de las médias (análise de variancia) dei perimetro braquial entre los diferentes trimestres dei embarazo, mostró que no hay diferencia estadisticamente significativa $(\mathrm{P}>0,05)$.EI análisis de correlación de Pearson mostró que, ei perimetro braquial y el peso de la mujer estan fuertemente correlacionados en cualquier periodo de la gestación. Por otro lado, ei perimetro braquial no estuvo correlacionado con la edad gestacional, altura uterina y altura de la mujer.Se concluye que ei perimetro braquial es un indicador relativamente estable durante ei embarazo e independiente de la edad gestacional, portanto puede ser medido en cualquier época dei embarazo para estimar ei peso pré-gestacional y de esta manera reflejar ei estado nutricional previo y/o actual de la gestante.
\end{abstract}

Descritores: antropometria, perimetro braquial, gestante, nutrición.socialização.

\section{INTRODUCCIÓN}

La antropometría es uno de los métodos más utilizados para determinar la composición corporal, una estimación del estado nutricional de individuos o poblaciones, puede ser obtenida a travez de la medición de varios indicadores antropométricos (peso, altura, perímetro braquial, pliegues cutáneos entre otros), que además de ser fáciles de medir permiten ahorrar e uso de métodos de laboratorio, que son mas caros y trabajosos.
El uso de indicadores antropométricos ha sido extensamente aplicado en niños y adultos en general para diagnóstico de estado nutricional y últimamente el interés está en el grupo de mujeres gestantes. Sin embargo, aun existe la preocupación del uso de tecnologias más simples, de bajo costo, buena reproducibilidad y de fácil aplica-ción. La medida del perímetro braquial (PB) satisface estas condiciones, y actualmente su uso es recomendado en mujeres gestantes (SHAH, 1982; HUSAINI,1986; ANDERSON, 1989; KRASOVEC, 1989 e 1991).

\footnotetext{
Publicación preparada con datos de la tesis de Maestria realizada en la Facultad de Salud Pública de la Universidad de São Paulo (FSPUSP), Brasil.

1 Pediatra, Magister en Salud Pública. Alumna de post graduación en nivel de doctorado, Departamento de Salud MaternoInfantil,. Facultad de Salud Pública, Univeridad de São Paulo.

2 Profesor Principal en el Departamento de Salud Materno-Infantil, Facultad de Salud Pública, Univesidad de São Paulo. Dirección postal: Departamento Materno-lnfantil. Faculdade de Saúde Pública, Universidade de São Paulo, Av. Dr. Arnaldo 715, $2^{\circ}$ andar, sala 218, São Paulo - SP - CEP: 01246-904, Fone: (011) 3061-5233 R 7702/7703 Fax: (011) 853-0240.
} 
Diferentes puntos de corte del PB mostraron buena capacidad para diagnosticar a mujeres con riesgo de tener um recién nacido de bajo peso (KRASOVEC \& ANDERSON, 1991), en comparación con resultados obtenidos por otros métodos antropométricos clásicos. Probablemente, las diferencias respecto al nivel de corte se deban al hecho de que las investigaciones que proponen el uso del PB, fueron realizadas en poblaciones con realidad nutricional diferente.

A pesar de los resultados importantes sobre el PB como indicador de estado nutricional materno (LECHTIG, 1988; SHAH, 1991), todavía existen dudas sobre la aplicación de este indicador en el diagnóstico de estado nutricional de la embarazada, debido a las controversias en relación al comportamiento de esta medida durante el embarazo (MERCHANT, 1989; LILJESTRAND, 1991), así como sobre el punto de corte utilizado para tal diagnóstico.

Por esta razón se propuso el presente estudio para investigar este indicador en embarazadas residentes en la ciudad de Sao Paulo-Brasil, y verificar su relación con otras medidas antropométricas, así como el comportamiento de esta medida en el transcurso del embarazo.

\section{MATERIAL Y MÉTODOS}

Fueron escogidas dos unidades de salud de la región de Butantã (C. S. Vila São Luis y Hospital Universitário) en la zona oeste de la ciudad de São Paulo para realizar el estudio, constituyéndose una muestra no probabilística de 200 gestantes, en función del acceso de las mismas al servicio de prenatal. Dichas gestantes fueron atendidas durante el período de mayo de 1991 a febrero de 1992.

Las embarazadas incluidas tenían las siguientes características: a) mujeres mayores de 15 años que estuviesen inscritas en el servicio de pre natal y cuya primera consulta hubiese ocurrido hasta la vigésima semana del embarazo, b) que no hayan registrado ninguna patología diagnosticada como propia o asociada al embarazo, c) que no hayan presentado embarazo múltiple.

La gestantes fueron entrevistadas y examinadas hasta obtener tres mediciones antropométricas, estableciendo un intervalo mínimo de cuatro semanas entre cada consulta pre natal. El peso, altura, altura uterina, y perímetro braquial fueron obtenidos de acuerdo a los padrones recomendados (WHO, 1983). La edad gestacional fue calculada a partir del último periodo menstrual y el peso pre gestacional fue obtenido por interrogatorio en la primera entrevista con la gestante.
Para el análisis de los datos fueron calculados los promedios de las variables estudiadas en los tres trimestres del embarazo, tomando como unidad de análisis a cada embarazada, obteniendo después, tres grupos independientes de embarazadas con sus respectivas medidas antropométricas.

Para comparar los promedios del perímetro braquial se procedió al análisis de varianza atravez del método ANOVA, entre los promedios obtenidos para cada gestante en los tres períodos diferentes del estudio y entre grupos de gestantes controlando edad gestacional (por trimestres). Para el estudio de correlación entre las variables antropométricas entre sí y con la edad gestacional fue utilizado el coeficiente de correlación de Pearson. Las diferencias entre las variables estudiadas fueron consideradas estadísticamente significativas cuando $\mathrm{p}<0,05$.

Los resultados obtenidos fueron confirmados utilizando otros modelos de selección; así por ejemplo, las medidas de las gestantes fueron agrupadas, tomando como unidad de observación el tiempo (momento en que fueron medidas) sin controlar edad gestacional, obteniendo de esta manera medidas en tiempo 1 , tiempo 2 y tiempo 3, en este modelo fue realizada la prueba t-Student para comparación de las medidas del perímetro braquial (datos no expuestos por no alterar las conclusiones del estudio). También fueron realizados los mismos ensayos/pruebas estadísticos con los datos de los casos que se perdieron en la última fase del estudio ( $\mathrm{N}=75)$, obteniendo los mismos resultados.

\section{RESULTADOS}

En la Tabla l (anexo) podemos observar los promedios alcanzados en las medidas antropométricas de las gestantes durante los tres trimestres del embarazo. Se puede observar que la ganancia de peso en función del peso pre gestacional (PPG) varió de 3,56 kg (para las mujeres en el primer trimestre) a 5,50 kg en el segundo trimestre y 7,65 para las del tercer trimestre. El promedio de la altura uterina también aumentó de acuerdo al avance de la edad gestacional, como se espera en una gestación normal. E1 promedio del PPG informado osciló entre 55,54 y 56,63 kg. Las medias del perímetro braquial son similares en los tres trimestres del embarazo (Tabla 1) y la diferencia entre los promedios del PB no es estadísticamente significativa (Tabla 2 - anexo).

E1 estudio de correlación entre el PB y las otras medidas antropométricas mostró que no existe asociación significativa entre el PB y edad ges- 
tacional ( $r=0,06$; 0,06; y 0,02 para los tres trimestres respectivamente); entre PB y altura materna ( $r=0,03$; 0,1 ; y 0,29 para el primer, segundo y tercer trimestre respectivamente) y entre el $\mathrm{PB}$ y altura uterina ( $\mathrm{r}=0,02 ; 0,1 ; \mathrm{y} 0,21)$ lo que sugiere que el PB es independiente de la edad gestacional (Tabla 3 - anexo). Por otro lado, entre el peso de la gestante y el PB se verifica una fuerte asociación ( $\mathrm{r}=0,77 ; 0,84 ;$ y 0,89$)$ en los tres trimestres del embarazo (Tabla 3).

\section{DISCUSIÓN}

E1 grupo de mujeres estudiadas obtuvo promedios antropométricos similares a los obtenidos en otros estudios realizados anteriormente en Brasil (SIQUEIRA, 1975; DESAI,1980). Observese que los promedios alcanzados en el presente estudio, para los parámetros antropométricos, corresponden a las referencias de mujeres brasileñas consideradas saludables, no desnutridas ni obesas (SIQUEIRA, 1979).

En relación a la ganancia de peso en el primer trimestre en función del PPG, informado, se verificó un aumento promedio de $3,56 \mathrm{~kg}$. Si aceptamos que en el primer trimestre del embarazo, la ganancia de peso está aproximadamente entre 1-2 kg. (HITTEN Y LEITCH,1971), entonces las embarazadas de este grupo tuvieron una ganancia ponderal de 2,06 kg. más de lo esperado. Si son gestantes consideradas normales y no existe otro factor que explique esa ganancia mayor de peso, entonces devemos sospechar que la información del peso pre gestacional es imprecisa.

Por otro lado, si llevamos en consideración que el incremento del peso para un embarazo normal debería ser 20\% de su PPG, esto significaría que estas embarazadas deberían ganar durante todo el embarazo um promedio ponderal de $11,22 \mathrm{~kg}$., y que de estos apenas 1,7\% corresponderían al primer trimestre (HITTEN \& LEITCH, ] 971). ¿Como se explica entonces los 2,06 kg a más que significarían 18,5\% de ganancia de peso para el primer trimestre?

Estos resultados nos permiten tornar discutible el uso del PPG como punto de partida en las curvas de evaluación de la ganancia de peso de la embarazada.

Sin embargo, el PPG es un indicador de estado nutricional muy importante para predecir resultado del embarazo, como ya fue demostrado en varios estudios clásicos como los de SMITH, 1947; ANTONOV, 1947; STEIN, 1972; NAEYE, 1973, entre otros. La precisión y confiabilidad de esta medida es entorpecida por la estimación teórica y/o información de la propia embarazada, por lo que se hace necesario investigar otros indicadores que representen el PPG.

Se puede decir que el perímetro braquial es un indicador relativamente estable en el embarazo ya que los cambios que podrían haber ocurrido en cada mujer no interfirieron para una tendencia gradual en un solo sentido, indicando aumento o disminuición de la medida durante el embarazo. E1 hecho de no estar correlacionado con la edad gestacional ni con la altura uterina refuerza la premisa de independencia y estabilidad del PB durante el embarazo.

Las correlaciones observadas en el presente estudio entre PB y peso de la gestante, también fueron observadas en investigaciones anteriores (ANDERSON, 1989; KRASOVEC, 1989; OLUKOYA, 1991). Otros estudios realizados en gestantes confirmaron que el PB de las desnutridas es menor que el de las normales (TIBREWALA \& SHAH, 1978), y el de las normales es menor que el de las obesas, cuando estas fueron clasificadas de acuerdo al índice peso/altura por edad gestacional (SIQUEIRA, 1979). Ya que valores elevados de PB corresponden a valores elevados de peso e viceversa, podemos sustentar que una medida de PB podría substituir la información del peso informado y/o estimado, como es el caso del peso pro gestacional.

\section{CONCLUSIONES Y RECOMENDACIONES}

La medida del perímetro braquial durante el embarazo no se modifica con el aumento de la edad gestacional, por lo tanto, es una medida estable durante la gestación.

E1 peso y el perímetro braquial de la gestante están fuertemente correlacionados en los tres trimestres del embarazo.

Devido al carácter de independencia del perímetro braquial y a su relación con el peso de la embarazada, puede considerarse el PB como um potencial indicador del peso pre gestacional y del peso actual de la gestante.

Se sugere la elaboración de un instrumento que utilice el perímetro braquial, asociado o no a otro indicador para diagnóstico de estado nutricional de la embarazada, sustituyendo el peso pre gestacional.

Es necesario realizar investigaciones para determinar el valor predictivo del perímetro braquial en la evaluación de la desnutrición en gestantes, procurando determinar los niveles adecuados de corte, para mujeres con diferente composición corporal en estado de gravidez y de no gravidez. 


\begin{abstract}
Weight, height, arm circumference and uterine height during pregnancy were compared in a cross sectional study with two hundred pregnant women, in order to determine the relationship between maternal arm circumference and other anthropometric indicators, and to determine patterns of changes of arm circumference during pregnancy. During the study, maternal arm circumference remained stable in the three trimesters of pregnancy. An analysis of arm circumference means showed no statistically significant diferences $(P>0,05)$.Pearson correlation analysis showed that arm circumference was strongly correlated with weight at any time during pregnancy; on the other hand, it was not correlated with gestational age, maternal height or uterine height at different trimesters. This implies that a woman's arm circumference can be measured at any point in pregnancy to estimate pre-pregnancy weight. These findings, together with the practical advantages of using maternal arrn circu ference, considerably strengthen the argument for using arm circumference for nutritional monitoring of pregnant women.
\end{abstract}

Key-words: anthropometry, mid arm circumference, pregnant women, nutrition.

\section{REFERÊNCIAS BIBLIOGRÁFICAS}

ANDERSON, M. A. The relationship between maternal nutrition and child growth in rural India, 1989. [PH. D Dissertation, Tufts University].

ANTONOV, A. N. Children born during the siege of Leningrad in 1942. J. Pediatr., 30: 250-59, 1947.

DESAI, I. D. et al. Food habits and nutritional status of agricultural migrant workers in southern Brazil. Am. J. Clin. Nutr, 33: 702-714, 1980.

HUSAINI, Y. K. et al. Maternal malnutrition, outcome of pregnancy, and a simple tool to identify women at risk. Food and Nutr Bull., 8 (1): 71 -78, 1986.

HYTTEN, F.; LEITCH, I. The phisiology of human pregnancy. $2^{\text {nd }}$ ed. London, Oxford University Press,1971.

KRASOVEC, K. An investigation lnto the use of maternal arm circumference for nutritional monitoring of pregnant women, 1989. [SC. D. Dissertation, Johns Hopkins Universlty School of Higiene and Public Health].

KRASOVEC, K. Arm circumference, background issues, chapter $10 \mathrm{In}$ : KRASOVEC, K; ANDERSON, M. A. edit. Maternal nutrition and pregnancy outcornes: anthropometric assesment. Washington. Pan American Health Organization, PAHO, scientific publication n.529, 1991.

KRASOVEC, K.; ANDERSON, M. A. Maternal nutrition and pregnancy outcomes: anthropometric assesment. Washington. Pan American Health Organization, PAHO Scientific $\mathrm{Pu}-$ blication,529, 1991.

LECHTIG, A. Predicting risk of delivering low blrth welght bables: wlch lndlcator ls better? J. Trop. Pediatr, 34: 34-41, 1988.
LILJESTRAND, J.; BERGSTROM, S. Antenatal nutrltlonal assesment: the value of upper arm clrcunference. Gynecolo. Obstet. Invest., 32: 81-3, 1991.

MERCHANT, K. et al. The use of upper arm anthropometry to predict fetal growth ln a bollvlan populatlon. Am. J. Fhys.Anthropol., 78 (2), 1989. (Abstract).

NAEYE, R. L. et al. Effects of maternal nutrltion on the human fetus. Pediatrics, 52: 494503, 1973.

OLUKOYA, A. A.; GIWA, O. F. Maternal weight and weight gain during pregnancy - can the arm circunference be used as surrogate?. Afr. J. Med Sci, 20: 155-62, 1991.

RICALDE, A. E. Contribuição para o estudo do perímetro braquial na avaliação do estado nutricional da gestante. Sao Paulo, 1994. [Dissertaçao de Mestrado - Faculdade de Saúde Pública].

SHAH, A. Apropriate technology and perinatal cate: The Lasa experience. Adv. Int. Maternal Child Health, 2: 1-15, 1982.

SHAH, K. The evolution of the use of arm circumference for assesing maternal nutritional status. In: KRASOVEC, K; ANDERSON, M. A. edit. Maternal nutrition and pregnancy outcomes: anthropometric assesment. Washington. Pan American Health Organization, PAHO, scientific publication n. 529, 1991.

SIQUEIRA, A. A. F. de et al. The influence of maternal height and weight gain and gestational age on the newborn's weight. Rev. SaúdePubl.. 9: 331-342,1975.

SIQUEIRA, A. A. F. Estudo de um conjunto de curvas antropométricas no diagnóstico de estado nutricional e sua relaçao com o tamanho do recém- nascido. São Paulo, 1979. [Tese de Doutorado - Faculdade de Saúde Pública - USP]. 
SMITH, C.A. Effects of maternal nutrition upon the newborn infant in Holland (1944- 1945). J. Pediatr., 30: 229-3, 1947.

STEIN, Z. et al. Nutrition and mental perfonnance. Science, 178: 708-13, 1972.

TIBREWALA, S.; SHAH, K. The use of arm circumference as an indicator of body weight in adult women. Baroda J. Nutr. 5: 43-45, 1978.
WORLD HEALTH ORGANIZATION. Measuring changes in nutritional status, Geneva, 1983. (WHO offset publication 39).

Recebido em 13/01/97

Aprovado em: 03/03/97

\section{ANEXO}

Tabla 1. Promedio y desvio padrón (dp) de las variables antropométricas de 200 gestantes en los tres trimestres del embarazo. São Paulo-Brasil, 1992.

\begin{tabular}{ccccccc}
\hline $\begin{array}{c}\text { Trimestre } \\
\text { del } \\
\text { embarazo }\end{array}$ & $\begin{array}{c}\text { Edad } \\
\text { gestadonal } \\
\text { (semanas) }\end{array}$ & $\begin{array}{c}\text { Altura } \\
\text { uterina } \\
(\mathbf{c m})\end{array}$ & $\begin{array}{c}\text { Perimetro } \\
\text { braquial } \\
(\mathbf{c m})\end{array}$ & $\begin{array}{c}\text { Peso } \\
(\mathrm{kg})\end{array}$ & $\begin{array}{c}\text { Peso pre- } \\
\text { gestadonal* } \\
(\mathrm{kg})\end{array}$ & Altura* $^{*}$ \\
\hline I & $10.53(2.20)$ & $6.8(6.4)$ & $27.36(2.25)$ & $59.10(7.49)$ & $55.54(6.70)$ & $156.21(5.40)$ \\
II & $20.63(3.88)$ & $19.9(5.1)$ & $27.31(3.73)$ & $61.70(11.79)$ & $56.20(10.60)$ & $157.1(6.20)$ \\
III & $30.62(1.82)$ & $29.4(2.7)$ & $27.49(2.80)$ & $64.28(8.81)$ & $56.63(8.03)$ & $156.8(5.30)$ \\
\hline
\end{tabular}

* Estas variables fueron obtenidas una única vez en la primera consulta pre natal.

Tabla 2. Análisis de varianza de los promedios del perimetro braquial en cada embarazada por tiempo de medición y por trimestre del embarazo $(n=200)$.

\begin{tabular}{cccc}
\hline $\begin{array}{c}\text { Entre } \\
\text { los } \\
\text { tiempos* }\end{array}$ & 599 & Grados de libertad & P estatistica \\
\cline { 2 - 4 } & 199 & 1,506 & 0,226 \\
\hline $\begin{array}{c}\text { Entre } \\
\text { trimestres** }\end{array}$ & 199 & 0,03 & 0,968 \\
\hline
\end{tabular}

* La unidad de análises es la medida del perimetro braquial en los tres tiempos diferentes sin controlar edad gestacional

** La unidad de análisis es la embarazada, por tanto, el perímetro braquial está controlado por la

edad gestacional (trimestres del embarazo)

a. Estatistica no significativa al nível de $5 \%$ 
Tabla 3. Correlación del perímetro braquial con otras variables antropométricas de la gestante en los diferentes periodos del estudio*

\begin{tabular}{cccc}
\hline $\begin{array}{c}\text { Correlacionada com el } \\
\text { PB }\end{array}$ & $\begin{array}{c}\text { Coeficiente de } \\
\text { Correlacion }\end{array}$ & $\begin{array}{c}\text { Intervalo de confianza } \\
95 \%\end{array}$ & $\begin{array}{c}\text { Trimestre del } \\
\text { em barazo }\end{array}$ \\
\hline Edad gestacional & $\mathrm{r}=0.06$ & $-0.24-0.36$ & Primero \\
& $\mathrm{r}=0.06$ & $-0.12-0.23$ & Segundo \\
& $\mathrm{r}=0.02$ & $-0.34-0.31$ & Tercero \\
\hline Peso & $\mathrm{r}=0.77$ & $0.61-0.87$ & Primero \\
& $\mathrm{r}=0.84$ & $0.71-0.92$ & Segundo \\
& $\mathrm{r}=0.89$ & $0.84-0.92$ & Tercero \\
\hline Altura & $\mathrm{r}=0.03$ & $-0.27-0.33$ & Primero \\
& $\mathrm{r}=0.15$ & $-0.04-0.32$ & Segundo \\
& $\mathrm{r}=0.29$ & $-0.04-0.56$ & Tercero \\
\hline Altura uterina & $\mathrm{r}=0.02$ & $-0.22-0.18$ & Primero \\
& $\mathrm{r}=0.10$ & $-0.02-0.21$ & Segundo \\
& $\mathrm{r}=0.21$ & $0.07-0.33$ & Tercero \\
\hline
\end{tabular}

* Fuente Ricalde, A.E. (Tesis de Maestria - Universidad de São Paulo - Brasil, 1994)

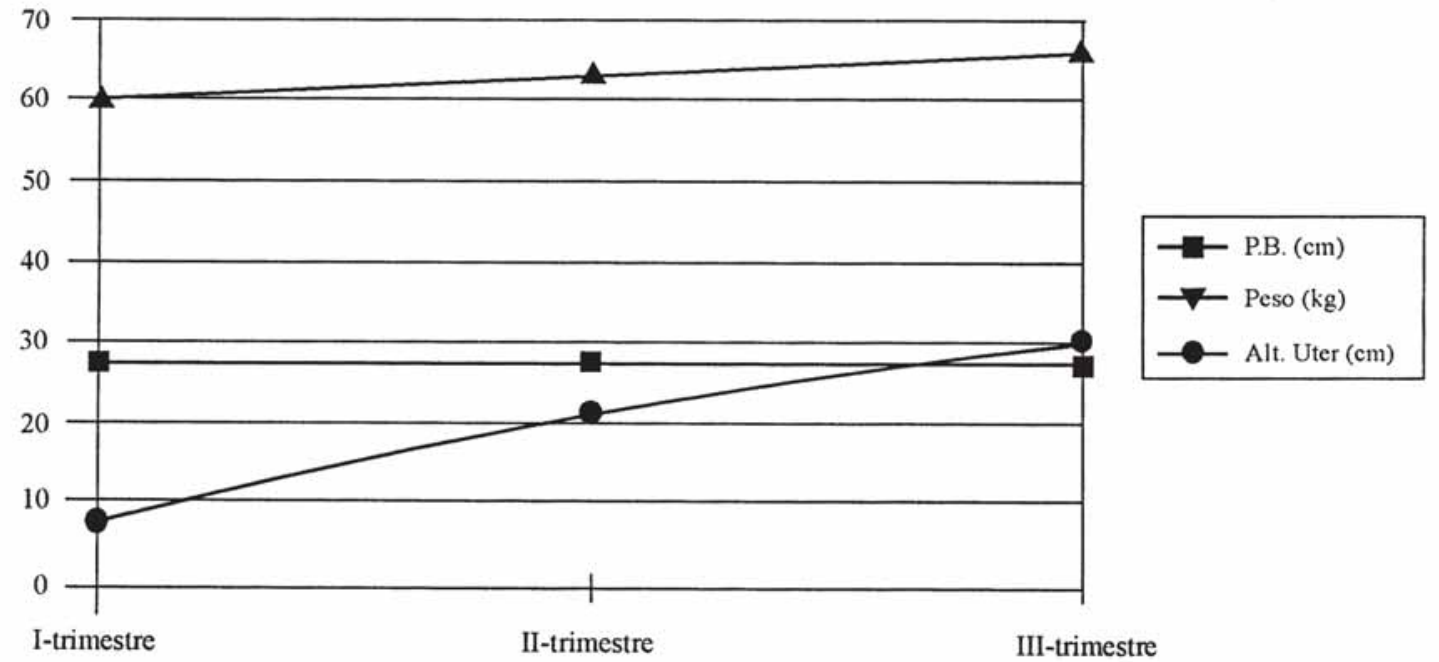

igura 1. Promedios del peso, altura uterina y perímetro braquial en los trimestres del embarazo $(\mathrm{N}=200)$ 